\title{
Meander-Line Polarizer for Omnidirectional Antenna
}

\author{
A. V. Stankovsky, S. V. Polenga, A. D. Nemshon, Y. A. Litinskaya, E. R. Gafarov, Yu. P. Salomatov \\ Institute of Engineering Physics and Radioelectronics \\ Siberian Federal University \\ Krasnoyarsk, Russia \\ e-mail: stankovskiy_a@mail.ru
}

\begin{abstract}
Meander-line polarizer for a half-wave vibrator is presented. Researched antenna was simulated and prototype was manufactured. The comparison of the theoretical and experimental data for investigated antenna (axial ratio) was produced. Influence of polarizer on half-wave vibrator matching characteristics was researched.
\end{abstract}

\section{Keywords — polarizer; meander-line; axial ratio.}

\section{INTRODUCTION}

Nowadays compact omnidirectional circularly polarized antenna is required for different tasks such as radio navigation, satelliteand wireless communications.Oneof the ways toobtainthe circular polarizationis the use ofpolarizers. The elementary polarizertypeconvenientforresearch, simulationandrealization is a meander-line structure. Smallthicknessofthe metallayer allows getting flat and thin polarizer. This design is especially suitable for half-wave vibrator antennas, where the polarizer shouldhave a cylindrical shape. Thin structure allows changing shape easily within certain limits.

Meander-line polarizer was described in the article [1], where possibility of cylindrical shape polarizercreation was researched, and the polarizer operationprinciplewas described. The dimensions for four-layer meander-line polarizers and axial ratio (AR) measurement results are shown in [1] also. Optimization of one-layer polarizer was produced by using presented approach and modern CAD software.

\section{MEANDER-LINEDESIGN}

Meander-line polarizer is a set of thin metal structures in meander form (Fig. 1). Its operating principle is detailed and calculation equation for polarizer sizes are presented in articles $[2,3]$.

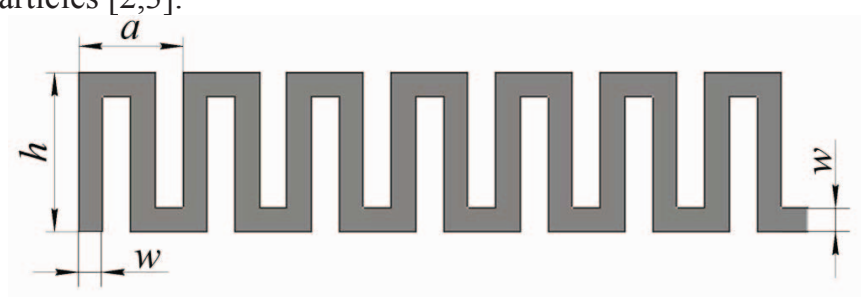

Fig. 1. Meander-line.

For flat polarizer realization, several meander-lines are located at a defined distance $(s)$ from each other in one plane, as shown in Fig. 2. The number of periods $(n)$ and number of meander-lines $(N)$ are chosen considering an illuminator sizes and a distance from illuminator to polarizer.

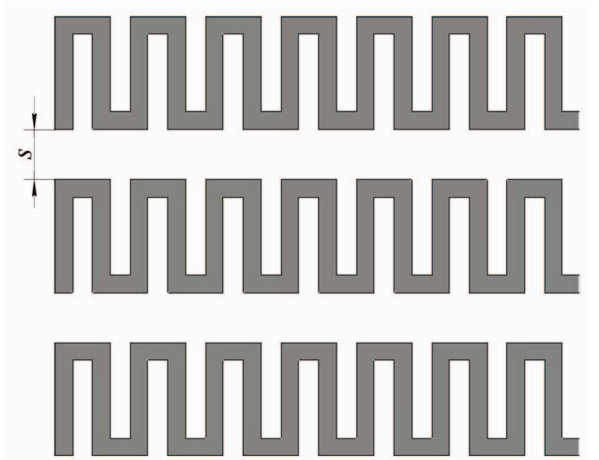

Fig. 2. Plane meander-line polarizer.

The calculated sizes of the meander-line and polarizer have been optimized for one-layer operating at central frequency of 2.4-2.5 GHz band. One-layer polarizer can be used for a narrow-band applications, and multilayer polarizers allow to increase operation frequency band as described below in chapter IV.

\section{MEANDER-LINEPOLARIZER DESIGN}

For cylindrical polarizer the supporting cylinder of required radius was made of foamed polystyrene. This material has dielectric constant $\varepsilon \approx 1.5$. The dielectric permittivity does not influence the antenna and polarizer characteristics as experimental results show. The structure of several meander-lines was fabricated of the copper-foil deposited polyethylene terephthalate (PET) $0.25 \mathrm{~mm}$ thick. This material is flexible, that allows getting any required shape. The meander-line structure is inclined at angle $45^{\circ}$ for left-hand or $-45^{\circ}$ for right-hand circular polarization and is wrapped around dielectric cylinder (Fig. 3).

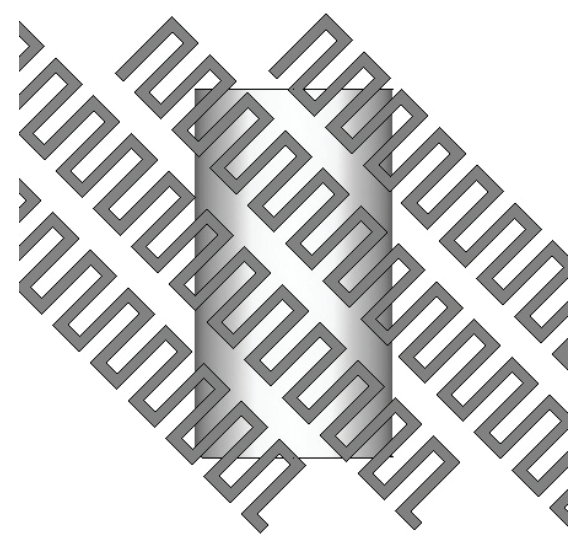

Fig. 3. Plane meander-line polarizer. 
A half-wave electric vibrator is used aspolarizer illuminator and is placed in the cylinder center. Vibrator produces the torus shape radiation pattern (RP). Thus, polarizer is supposed to be placed at the same distance from the antenna in all directions. The distance between the meander-lines and their number aren't limited in a flat polarizer (at a known size $h$ ). For cylindrical polarizer these parameters directly depend on cylinder circumference (diameter $D$ ). Thus, the distance between meander-lines $(s)$ is calculated as follows:

$$
s=\sqrt{\left(\frac{D \cdot \pi}{N}\right)^{2} \frac{1}{2}}-h .
$$

The presented equation takes into account that distance between the last and the first meander lines will be equal to $s$ at structure wrapping on the cylinder. The polarizer prototype was fabricatedaccording to calculations results (Fig.4).

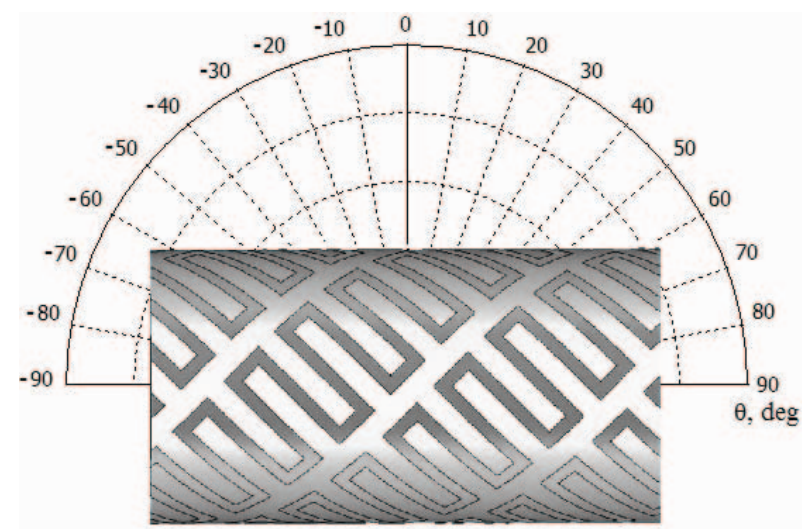

Fig. 4. Cylindrical meander-line polarizer.

\section{RESUltsand Discussion}

The following characteristics are obtained after numerical parameters optimization (Fig.5-8).

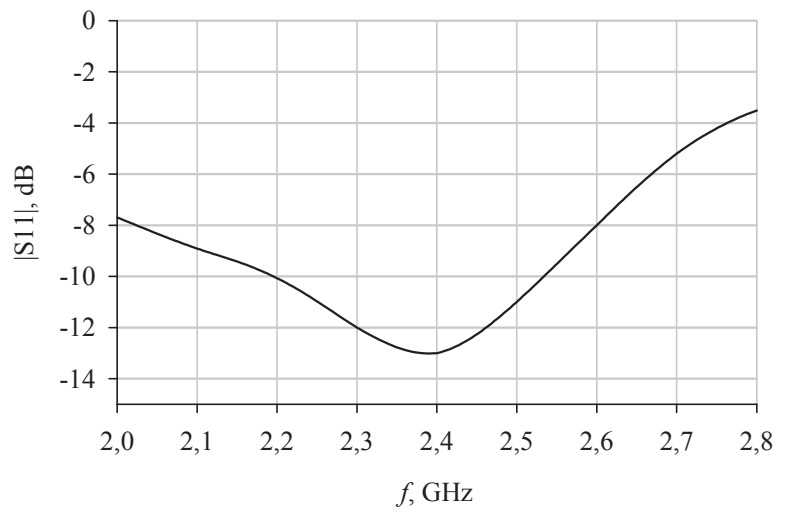

Fig. 5. Calculated $S_{11}$ parametrs of vibrator antenna with a polarizer.

The vibrator antenna with a polarizer isproperly matchedwithin necessary frequency range. $S_{11}$ parameter of the same vibrator antennawithout polarizer ispresented in Fig.6.

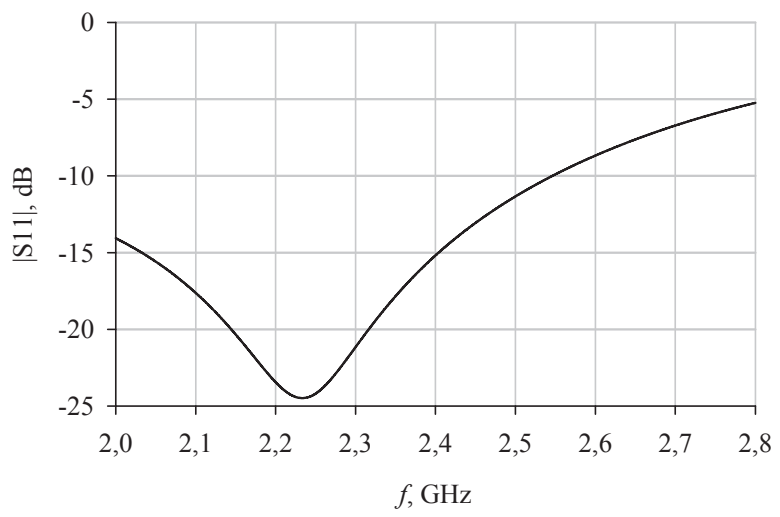

Fig. 6. Calculated $S_{11}$ parameter of vibrator antenna without a polarizer.

The vibrator antenna without a polarizer has bettermatching characteristics, but the central working frequency is $2.245 \mathrm{GHz}$.The polarizer shifts working frequency range.However, prototype of antenna with a polarizer providesbetter matching than CAD model.

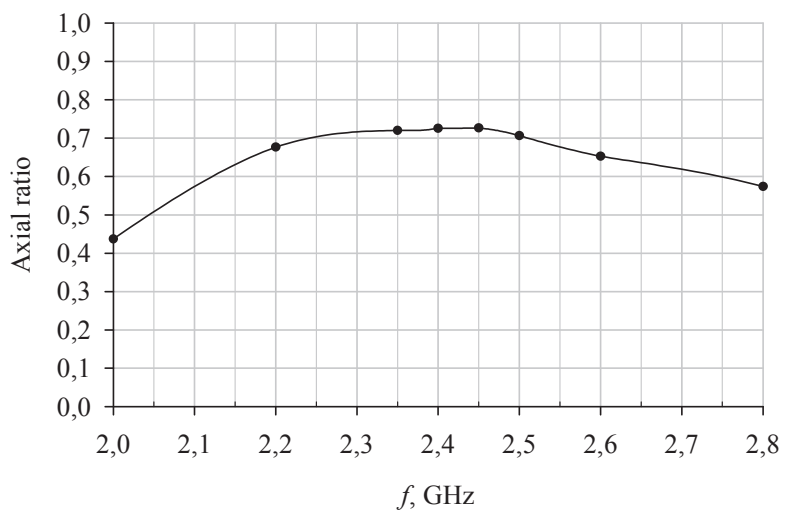

Fig. 7. Calculated axial ratio of vibrator antenna with a polarizer.

Axial ratio corresponding toradiation pattern maximumis not less than 0.7 within the working frequency range, i.e. the cross-polarization level is less than $-14.5 \mathrm{~dB}$ (Fig.8).

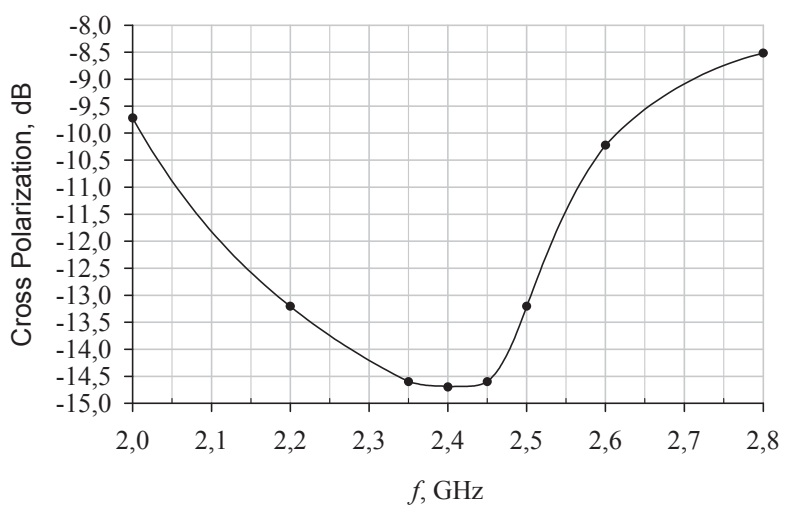

Fig. 8. Calculated cross polarization of vibrator antenna with a polarizer

As already told in chapter II, it is possible to expand a working frequency range by using several meander-line polarizers, placed one above other (Fig.9). 


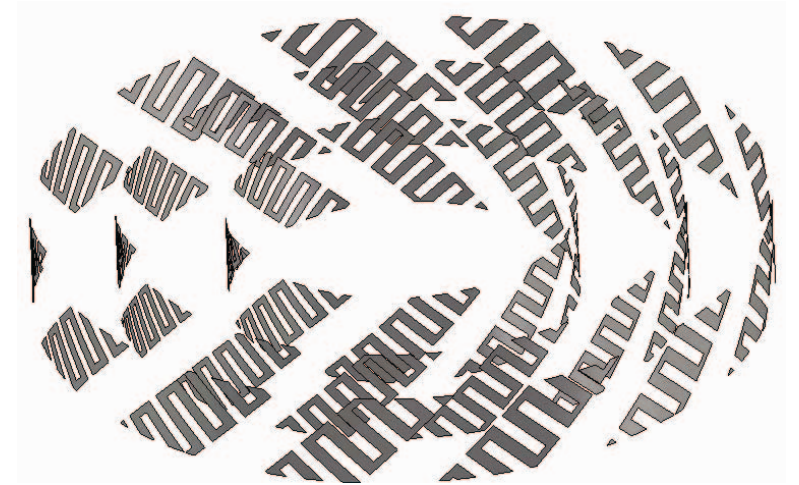

Fig. 9. Segment of 3-layer polarizer structure.

Axial ratio of circular three-layer polarizer is shown in Fig. 10 .

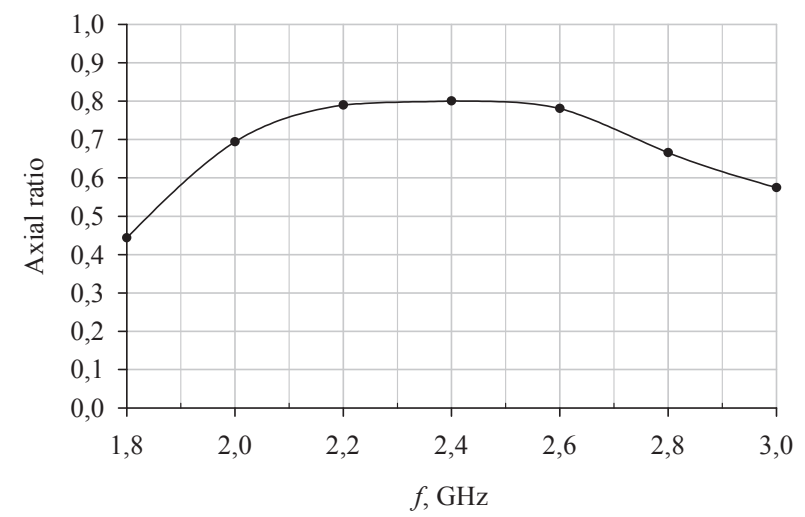

Fig. 10. Calculated axial ratio of vibrator antenna with a three-layer polarizer

Several layers polarizer allows to expand the working frequency range up to more than $30 \%$ and to increaseaxial ratio for center frequencyup to 0,8.Cross-polarization level is as low as $-19 \mathrm{~dB}$ (Fig. 11).

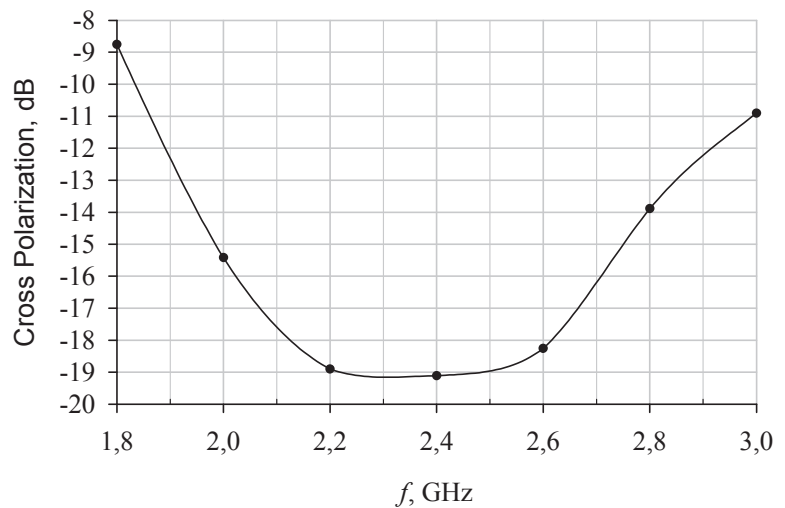

Fig. 11. Calculated cross polarization of vibrator antenna with a three-layer polarizer.

\section{MEASUREMENTS}

Based on the calculation resultstwo models of antennas with a polarizer for left and right circular polarizations were manufactured (Fig. 12).

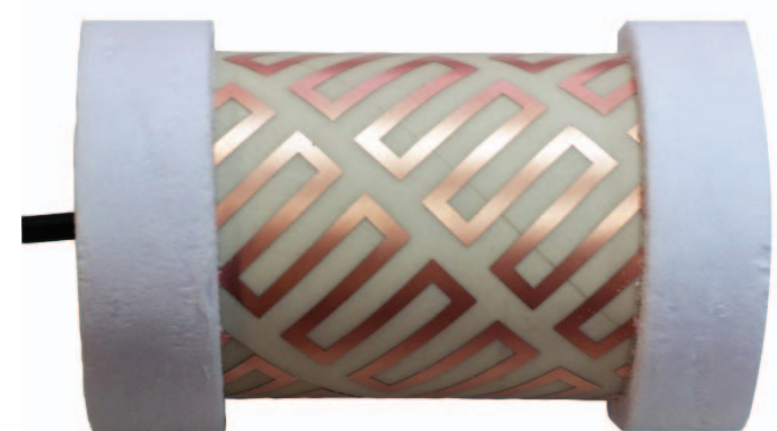

Fig. 12. Antenna prototype.

Cylinder dimensions are diameter $-65 \mathrm{~mm}$, length -120 $\mathrm{mm}$.

Measurements were carried out in anechoic chamber in far field. Results are presented in Fig.13-16.

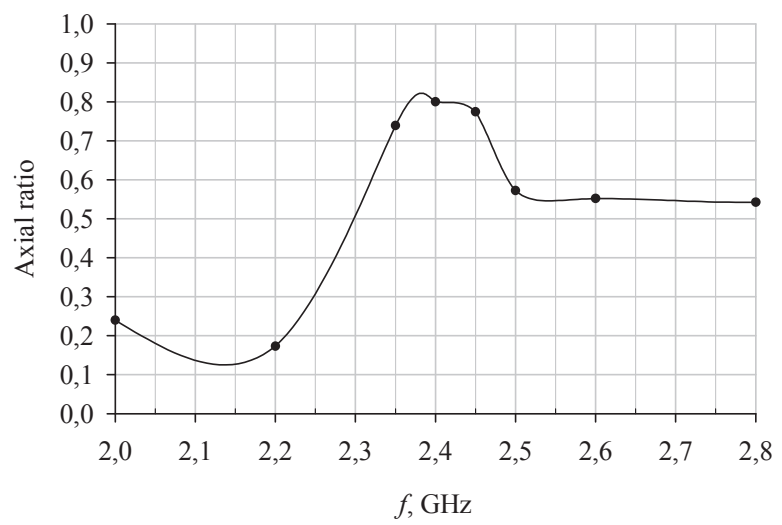

Fig. 13. Measured axial ratio of vibrator antenna with a polarizer (left-hand polarization).

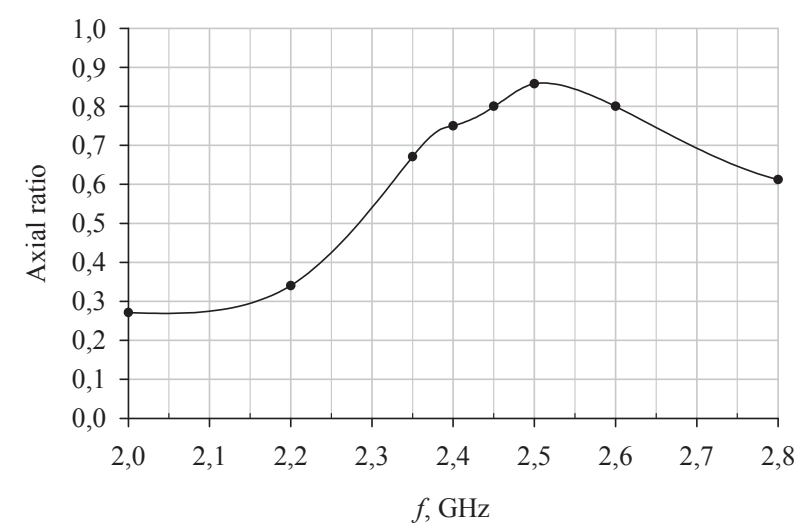

Fig. 14. Measured axial ratio of vibrator antenna with a polarizer (right-hand polarization).

Axial ratio measurement results differ from the simulation.This is partlyconnected to the materialslosses unaccountedin the calculations. Prototype manufacturing inaccuracy also influences antenna characteristics. The last fact explains differences between $\mathrm{AR}$ for the left and right 
polarizations.Nevertheless, axial ratio values in the working frequency rangearesimilar.

Axial ratio angular dependence at the central frequency $(2.445 \mathrm{GHz})$ is presentedin Fig.15. Angular scale corresponds to Fig.4.

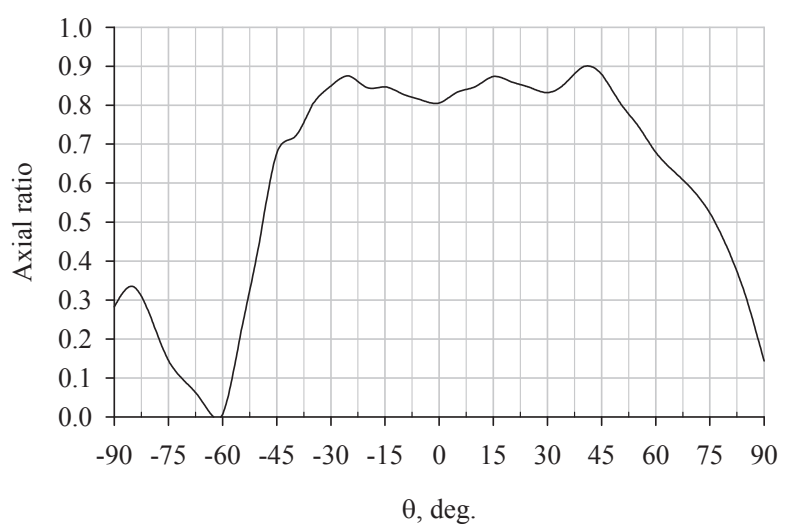

Fig. 15. Measured axial ratio angular dependence at $2.445 \mathrm{GHz}$.

Axial ratio value is stable in $\pm 45^{\circ}$ angle range. Meanderlines projection changes with angle increasing. This fact leads to significant axial ratio reduction at anglesmore than $45^{\circ}$. However, half-wave vibrator antenna employment makes no sense for directions $\theta> \pm 45^{\circ}$ due to low level of radiation pattern.

Radiating pattern of vibrator antenna with polarizer is insignificantly tilted due toasymmetry of half-wave vibrator feed point and feed line (Fig. 14).

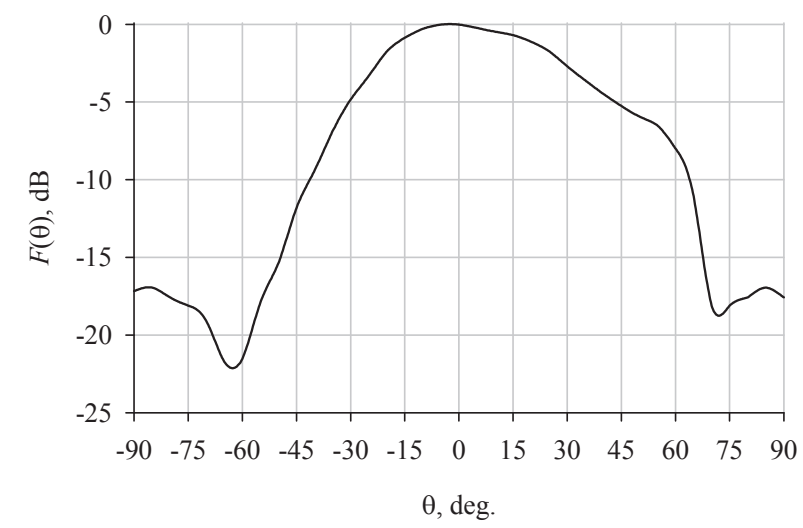

Fig. 16. Measured radiation patternof vibrator antenna with a polarizer at $2.445 \mathrm{GHz}$.

\section{CONCLUSION}

Presented polarizer type can be used together with half wave vibrator antennastotransform linear polarization to circular. Meander-lines arrangement relative to the antenna allows producing of both right-hand and left-hand circular polarizations.Results of modeling and experimental measurements demonstratethe possibility of production and practical application of such polarizer.

\section{References}

[1] Leo Young, Lloyd A. Robinson, Colin A. Hacking. Meander-Line Polarizer// 1973 IEEE Transactions on Antennas and Propagation, May 1973.

[2] Ruey-Shi Chu, Kuan-Min Lee. Analytical Model of a Multilayered Meander-Line Polarizer Plate with Normal and Oblique Plane-Wave Incidence // 1987 IEEE Transactions on Antennas and Propagation, vol. AP-35, No. 6, June 1987. P. 652-661.

[3] Marc-André Joyal, Mathieu Riel, Yves Demers, Jean-Jacques Laurin. A Meander-Line Circular Polarizer Optimized for Oblique Incidence// 2015IEEE Transactions on Antennas and Propagation, vol. 63, No. 12 December 2015. P. 5391-5398. 